\title{
Biallelic loss-of-function variants in PLD1 cause congenital right-sided cardiac valve defects and neonatal cardiomyopathy
}

\author{
Najim Lahrouchi, ${ }^{1}$ Alex V. Postma, ${ }^{2,3}$ Christian M. Salazar, ${ }^{4}$ Daniel M. De Laughter, ${ }^{5}$ Fleur Tjong, ${ }^{1}$ Lenka Piherová, ${ }^{6}$
} Forrest Z. Bowling, ${ }^{7}$ Dominic Zimmerman, ${ }^{1}$ Elisabeth M. Lodder, ${ }^{1}$ Asaf Ta-Shma, ${ }^{8}$ Zeev Perles, ${ }^{8}$ Leander Beekman, ${ }^{1}$ Aho Ilgun, ${ }^{3}$ Quinn Gunst, ${ }^{3}$ Mariam Hababa, ${ }^{1}$ Doris Škorić-Milosavljević, ${ }^{1}$ Viktor Stránecký, ${ }^{6}$ Viktor Tomek, ${ }^{9}$ Peter de Knijff, ${ }^{10}$ Rick de Leeuw, ${ }^{10}$ Jamille Y. Robinson, ${ }^{11}$ Sabrina C. Burn, ${ }^{12}$ Hiba Mustafa, ${ }^{12}$ Matthew Ambrose, ${ }^{13}$ Timothy Moss, ${ }^{14}$ Jennifer Jacober, ${ }^{15}$ Dmitriy M. Niyazov, ${ }^{15}$ Barry Wolf, ${ }^{16,17}$ Katherine H. Kim, ${ }^{16,17}$ Sara Cherny, ${ }^{17,18}$ Andreas Rousounides, ${ }^{19}$ Aphrodite Aristidou-Kallika, ${ }^{20}$ Ceorge Tanteles, ${ }^{21,22}$ Bruel Ange-Line, ${ }^{23,24}$ Anne-Sophie Denommé-Pichon, ${ }^{23,24}$ Christine Francannet, ${ }^{25}$ Damara Ortiz, ${ }^{26}$ Monique C. Haak, ${ }^{27}$ Arend D.J. Ten Harkel, ${ }^{28}$ Cwendolyn T.R. Manten, ${ }^{29}$ Annemiek C. Dutman, ${ }^{30}$ Katelijne Bouman, ${ }^{31}$ Monia Magliozzi, ${ }^{32}$ Francesca Clementina Radio, ${ }^{32}$ Gijs W.E. Santen, ${ }^{33}$ Johanna C. Herkert, ${ }^{31}$ H. Alex Brown, ${ }^{11}$ Orly Elpeleg, ${ }^{34}$ Maurice J.B. van den Hoff, ${ }^{3}$ Barbara Mulder, ${ }^{1}$ Michael V. Airola, ${ }^{7}$ Stanislav Kmoch, ${ }^{6}$ Joey V. Barnett, ${ }^{11}$ Sally-Ann Clur, ${ }^{35}$ Michael A. Frohman, ${ }^{4}$ and Connie R. Bezzina ${ }^{1}$

'Amsterdam UMC, University of Amsterdam, Heart Center, Department of Clinical and Experimental Cardiology, Amsterdam Cardiovascular Sciences, ${ }^{2}$ Department of Clinical Cenetics, and ${ }^{3}$ Department of Medical Biology, Amsterdam UMC, Amsterdam, Netherlands. ${ }^{4}$ Department of Pharmacological Sciences and Graduate Program in Molecular and Cellular Pharmacology, Stony Brook University, Stony Brook, New York, USA. ${ }^{5}$ Department of Cell and Developmental Biology, Vanderbilt University School of Medicine, Nashville, Tennessee, USA. ${ }^{6}$ Research Unit for Rare Diseases, Department of Pediatrics and Adolescent Medicine, 1st Faculty of Medicine, Charles University and Ceneral University Hospital, Prague, Czech Republic. ${ }^{7}$ Department of Biochemistry and Cell Biology, Stony Brook University, Stony Brook, New York, USA. ${ }^{8}$ Department of Pediatric Cardiology, Hadassah, Hebrew University Medical Center, Jerusalem, Israel. ${ }^{9}$ Children's Heart Centre, 2nd Faculty of Medicine, Charles University in Prague, Motol University Hospital, Prague, Czech Republic. ${ }^{10}$ Department of Human Genetics, Leiden University Medical Centre, Leiden, Netherlands. "Department of Pharmacology, Vanderbilt University School of Medicine, Nashville, Tennessee, USA. ${ }^{12}$ Department of Obstetrics, Cynecology and Women's Health, ${ }^{13}$ Department of Pediatrics, Division of Pediatric Cardiology, and ${ }^{14}$ Department of Pediatrics, University of Minnesota, Minneapolis, Minnesota, USA. ${ }^{15}$ Department of Pediatrics, Ochsner Clinic, Tulane University, University of Queensland, New Orleans, Louisiana, USA. ${ }^{16}$ Division of Cenetics, Birth Defects and Metabolic Disorders, Ann and Robert H. Lurie Children's Hospital of Chicago, Chicago, Illinois, USA. ${ }^{17}$ Feinberg School of Medicine, Northwestern University, Chicago, Illinois, USA. ${ }^{18}$ Division of Cardiology, Ann and Robert H. Lurie Children's Hospital of Chicago, Chicago, Illinois, USA. ${ }^{19}$ Makarios Medical Centre, Nicosia, Cyprus. ${ }^{20}$ Ultrasound and Fetal Medicine Diagnostic Centre, Nicosia, Cyprus. ${ }^{21}$ Cyprus School of Molecular Medicine, Nicosia, Cyprus. ${ }^{22}$ Department of Clinical Cenetics, The Cyprus Institute of Neurology and Genetics, Nicosia, Cyprus. ${ }^{23}$ UMR 1231 INSERM, CAD, Université Bourgogne Franche-Comté, Dijon, France. ${ }^{24}$ Unité Fonctionnelle d'Innovation en Diagnostique Génomique des Maladies Rares, FHU-TRANSLAD, Centre Hospitalier Universitaire Estaing (CHU), Dijon Bourgogne, Dijon, France. ${ }^{25}$ Service de Génétique Médicale, CHU Estaing, Clermont-Ferrand, France. ${ }^{26}$ Medical Cenetics Department, UPMC Children's Hospital of Pittsburgh, Pittsburgh, Pennsylvania, USA. ${ }^{27}$ Department of Obstetrics and ${ }^{28}$ Department of Pediatric Cardiology, Leiden University Medical Centre, Leiden, Netherlands. ${ }^{29}$ Department of Obstetrics and ${ }^{30}$ Department of Pathology, Isala Women and Children's Hospital, Zwolle, Netherlands. ${ }^{31}$ University Medical Center Groningen, Department of Genetics, University of Groningen, Groningen, Netherlands. ${ }^{32}$ Cenetic and Rare Disease Research Division, Bambino Gesù Children's Hospital IRCCS, Rome, Italy. ${ }^{33}$ Department of Human Genetics, Leiden University Medical Center, Leiden, Netherlands. ${ }^{34}$ Department of Genetics, Hadassah, Hebrew University Medical Center, Jerusalem, Israel. ${ }^{35}$ Department of Pediatric Cardiology, Emma Children's Hospital, Amsterdam UMC, University of Amsterdam, Amsterdam, Netherlands.

Congenital heart disease is the most common type of birth defect, accounting for one-third of all congenital anomalies. Using whole-exome sequencing of $\mathbf{2 7 1 8}$ patients with congenital heart disease and a search in GeneMatcher, we identified 30 patients from 21 unrelated families of different ancestries with biallelic phospholipase D1 (PLD1) variants who presented predominantly with congenital cardiac valve defects. We also associated recessive PLD1 variants with isolated neonatal cardiomyopathy. Furthermore, we established that $\mathrm{p} .1668 \mathrm{~F}$ is a founder variant among Ashkenazi Jews (allele frequency of $\sim 2 \%$ ) and describe the phenotypic spectrum of PLD1-associated congenital heart defects. PLD1 missense variants were overrepresented in regions of the protein critical for catalytic activity, and, correspondingly, we observed a strong reduction in enzymatic activity for most of the mutant proteins in an enzymatic assay. Finally, we demonstrate that PLD1 inhibition decreased endothelial-mesenchymal transition, an established pivotal early step in valvulogenesis. In conclusion, our study provides a more detailed understanding of disease mechanisms and phenotypic expression associated with PLD1 loss of function.

Authorship note: NL and AVP are co-first authors and contributed equally to this work. SAC, MAF, and CRB jointly supervised the work.

Conflict of interest: The authors have declared that no conflict of interest exists.

Copyright: $\odot$ 2021, American Society for Clinical Investigation.

Submitted: July 9, 2020; Accepted: December 16, 2020; Published: March 1, 2021.

Reference information: J Clin Invest. 2021;131(5):e142148. https://doi.org/10.1172/JCl142148. 


\section{Introduction}

Congenital heart disease is the most common type of birth defect, accounting for one-third of all congenital anomalies, with a worldwide occurrence of 7 per 1000 live births (1). The majority of these defects include abnormalities of valve formation. Right-sided congenital heart disease includes abnormalities of the pulmonary and tricuspid valves, the right ventricle, and the right ventricular outflow tract. Recessive variants in PLD1, which encodes phospholipase D1, a signal transduction enzyme that hydrolyses the membrane lipid phosphatidylcholine to generate the lipid second messenger phosphatidic acid (2), were recently associated with severe right-sided congenital cardiac valve defects in 2 small families (3). Although no overt structural cardiac defects were noted, mice lacking PLD1 displayed moderately impaired function of the pulmonary and tricuspid valve on echocardiography (3). However, the functional consequences of PLD1 enzymatic activity and the mechanism by which PLD1 dysfunction leads to congenital valve abnormalities remain unknown.

Using whole-exome sequencing (WES) data from 2718 patients with congenital heart disease and a search in GeneMatcher (4), we identified 30 patients from 21 unrelated families of different ancestries with biallelic PLD1 variants. We established that p.I668F is a founder variant among Ashkenazi Jews. We expanded the phenotypic spectrum of PLD1-associated congenital heart defects and, for the first time to our knowledge, provide evidence that recessive variants in $P L D 1$ can also cause neonatal cardiomyopathy in the absence of congenital heart defects. Placement of the missense variants on the 3D crystal structure of PLD1, recently reported by several of the co-authors (5), localized them primarily to sites important for catalytic site integrity. Correspondingly, we uncovered a substantial loss of enzymatic activity through functional analysis. Finally, in line with a loss-of-function mechanism of PLD1, we demonstrate here that PLD1 inhibition decreased endothelial-mesenchymal transition (EndoMT), a pivotal early step in valvulogenesis (6).

\section{Results}

PLD1 variants cause congenital valvular defects or neonatal cardiomyopathy in multiple families. To uncover the genetic cause of severe right-sided congenital heart defects in a consanguineous Afghan family with 2 affected siblings (family A, Figure 1A), we performed WES for both parents and one of the affected siblings. In line with the expected recessive inheritance pattern, we identified a homozygous variant, p.R695C, in PLD1 (GenBank accession number: NM_002662) as the only cosegregating variant. We then searched for additional patients carrying biallelic variants in PLD1 by performing (a) WES for 75 patients with similar severe right-sided congenital heart defects from the National Registry of Congenital Heart Defects (CONCOR) in the Netherlands (7); (b) analysis of 2643 patients with congenital heart disease trios (i.e., the affected patient and both parents) who underwent WES from the Pediatric Cardiac Genomics Consortium (PCGC) $(8,9)$; and (c) a search using GeneMatcher (4). In total, we identified 30 patients from 21 unrelated families of different ancestries (Figure 1A, Table 1, and Supplemental Table 1) carrying either homozygous or compound heterozygous PLD1 variants. All 30 patients were diagnosed with severe congenital heart disease or cardiomyopathy at the fetal or neonatal stage.
Twenty-eight patients from 19 families (families A-S, Figure 1) presented with congenital cardiac valve malformations predominantly affecting the right side of the heart. These defects were characterized by severe tricuspid and/or pulmonary valve abnormalities (27 of 28), hypoplastic right ventricles (16 of 28), and/or hypoplastic pulmonary arteries (11 of 28) (Table 1, Supplemental Table 1, detailed phenotypic descriptions in Supplemental Note 1 , and echocardiography in a selection of patients in Supplemental Videos 1-4; supplemental material available online with this article; https://doi.org/10.1172/JCI142148DS1). Five of these 28 patients were diagnosed with Ebstein's anomaly. The extremely thin right ventricular wall in both patients from family A prompted a diagnosis of Uhl's anomaly, a rare heart defect characterized by a congenital partial or complete absence of right ventricular wall myocardium (Figure 1C and ref. 10). Two patients had dilated left ventricles and mitral regurgitation in addition to tricuspid and/or pulmonary valve defects. Six pregnancies were terminated because of a complex heart defect with poor prognosis (gestational age range, 13-22 weeks). One patient underwent an orthotopic heart transplantation at the age of 45 days because of worsening heart function (family S). Four patients survived less than 1 year as a result of their underlying structural heart defect. The majority of live-born infants required surgery in the first days or months of life, most commonly the placement of a Blalock-Taussig shunt and bidirectional Glenn palliation (Table 1).

Of note, 2 patients presented with severe isolated neonatal cardiomyopathy (i.e., in the absence of a structural congenital heart defect), a new finding for this syndrome (families $\mathrm{T}$ and $\mathrm{U}$, Figure 1 and Table 1). At 33 weeks of gestation, the patient from family $\mathrm{T}$ was found to have persistently reduced fetal movement. A fetal ultrasound revealed hydrops fetalis due to severe cardiomyopathy, and an emergency delivery by caesarean section was performed. Postnatal echocardiography showed severe systolic dysfunction of the left ventricle with severe mitral regurgitation (Supplemental Videos 5 and 6). Despite medical treatment, the child died on the third postnatal day as a result of intractable cardiac failure. The other patient (family $\mathrm{U}$ ) was a full-term neonate who developed difficulty breathing and bloody vomiting and died on postnatal day 8 . The autopsy showed sections of the heart with patchy replacement of the myocardium by histiocytoid cells and occasional plasma cells and eosinophils, a finding most consistent with histiocytoid cardiomyopathy. Both patients were screened for variants in approximately 300 cardiomyopathy-related genes and were found to be negative.

Overall, none of the patients who survived beyond infancy had dysmorphic features, intellectual disability, or notable developmental delays, suggesting that in the context of overt phenotypes, PLD1 loss of function (see below) is predominantly associated with isolated cardiac disease in humans.

We noted an autosomal-recessive mode of inheritance in all of the families and found that variants in $P L D 1$ were inherited from each of the unaffected parents. In total, we report 31 PLD1 variants, of which 21 are missense variants and 10 are expected to result in protein truncation (Supplemental Table 2). Two variants are predicted to affect splicing. Reverse transcription PCR (RT-PCR) analysis of mRNA isolated from a peripheral blood sample from the parents of family E confirmed mis-splicing that 
A

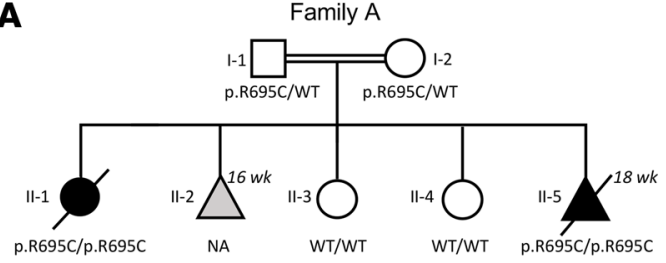

Family E

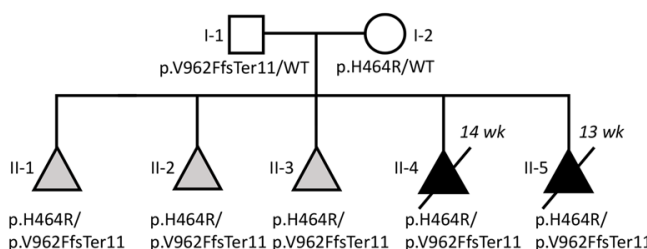

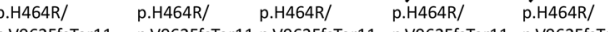

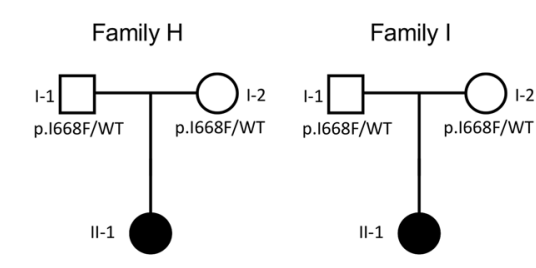

p. $1668 \mathrm{~F} / \mathrm{p} .1668 \mathrm{~F}$

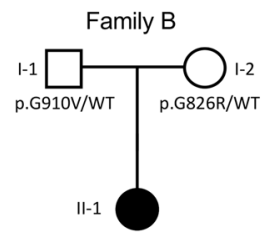

p.G826R/p.G910V

Family $\mathrm{F}$

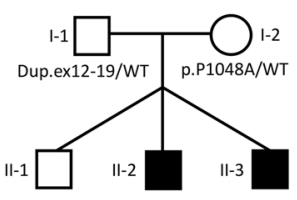

p.P1048A/ p.P1048A/ p.P1048A/ $\begin{array}{lll}\text { Dup.ex12-19 } & \text { Dup.ex12-19 } & \text { Dup.ex12-19 } \\ \text { D.P. } & & \\ & & \end{array}$

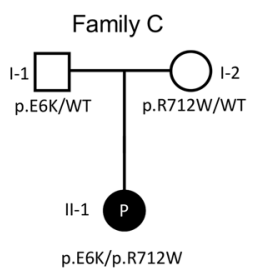

Family D

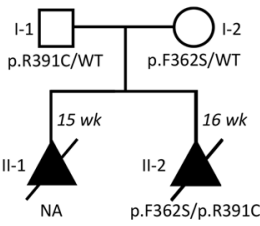

Family G

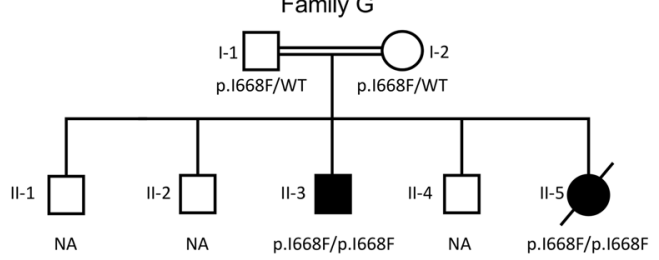

Family $\mathrm{N}^{*}$

Family

Family K

Family L

Family M
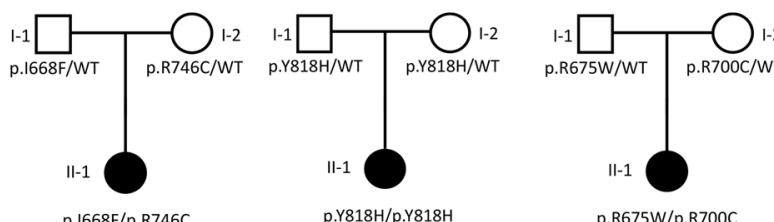

p.R675W/p.R700C

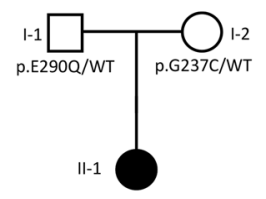

p.E290Q/p.G237C

Family $P$

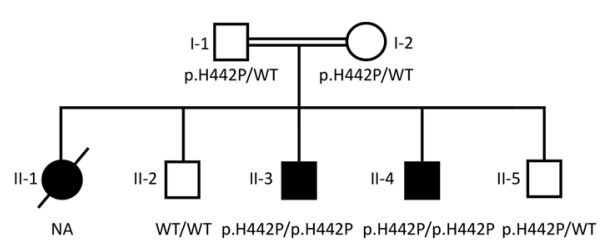

Family $\mathrm{O}^{*}$
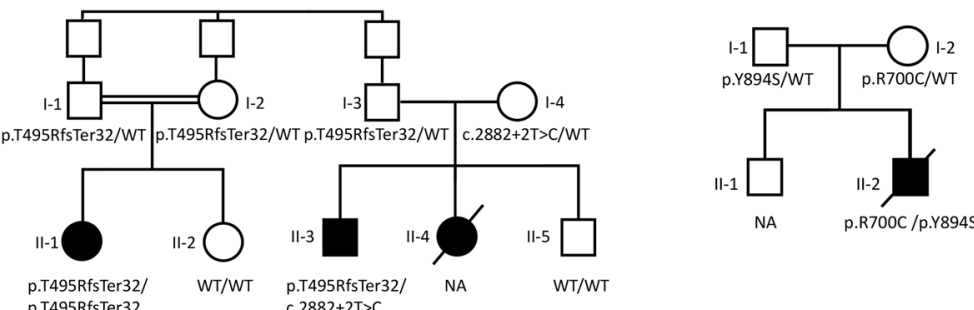

p.T495RfsTer32/ c. $2882+2 T>C$

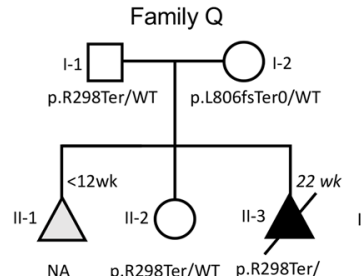

NA p.R298Ter/WT p.R298Ter/ p.L806fsTer0

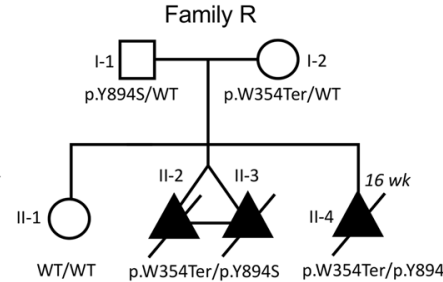

WT/WT p.W354Ter/p.Y894S p.W354Ter/p.Y894S

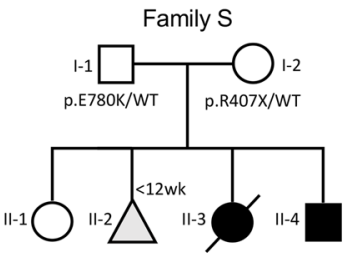

NA

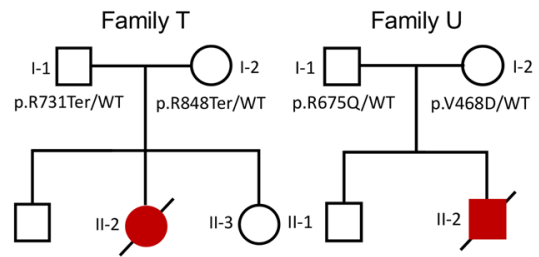

B
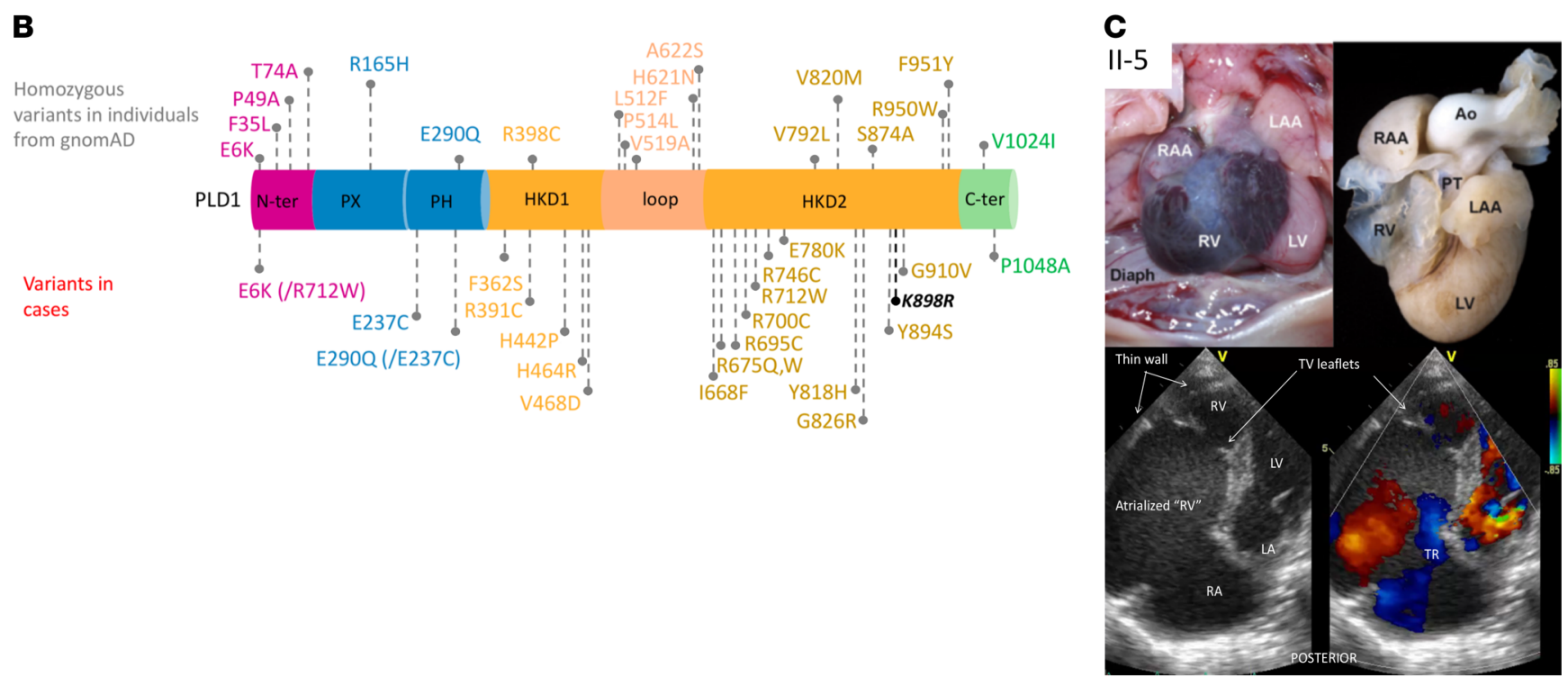
Figure 1. Recessive variants in PLD1 cause a spectrum of valvular congenital heart disease and neonatal cardiomyopathy. (A) Pedigrees. Solid symbols indicate the affected individuals (black indicates individuals with a congenital heart defect; red indicates individuals with fetal/neonatal cardiomyopathy), and symbols with a slash through them indicate deceased individuals. A double line indicates consanguinity. Males are indicated by squares and females by circles. Solid black triangles with a slash through them indicate fetal death or termination of pregnancy. Gray triangles indicate a miscarriage. Dup, duplication; ex, exon; fs, frame shift; NA, not available; Ter, termination; wk, age in weeks at termination of the pregnancy. "Previously published family (3). Families $\mathrm{H}-\mathrm{M}$ were identified through analysis of PCGC data, and pedigrees were not available $(8,9)$. (B) PLD1 domain structure and location of pathologic (bottom) and presumptive nonpathologic (top) missense variants. Black indicates an inactive allele (16) used as a baseline control. Gray dots, homozygous missense variants found in gnomAD individuals (11). Statistical comparison of loop variants in patients versus controls was performed using Fisher's exact test $(P=0.017)$. (C) Macroscopic appearance of the heart of fetus II-5 from family A. Upper left panel: Position of the heart in the thorax. Note the extremely dilated right ventricle with a thin translucent wall. A sharp demarcation can be seen between the abnormal right ventricular myocardium and normal left ventricular myocardium. Upper right panel: Anterior view of a formalin-fixed heart including large vessels, showing the paperthin right ventricular wall, which was partially collapsed because of tissue weakness. Bottom panels: Postnatal echocardiograms of child II-1 from family A displaying a thin-walled right ventricle with Ebstein's anomaly of the tricuspid valve and tricuspid regurgitation (see also Supplemental Video 1). This child also had pulmonary atresia. Ao, ascending aorta; Diaph, thoracic diaphragm; LA, left atrium; LAA, left atrial appendage; LV, left ventricle; $\mathrm{PT}$, pulmonary trunk; RAA, right atrial appendage; $\mathrm{RV}$, right ventricle; TV, tricuspid valve; TR, tricuspid regurgitation.

is predicted to result in a truncated PLD1 protein (Supplemental Figure 1). The duplication of exons $13-21$ found in family $F$ is predicted to result in a premature stop codon in exon 13. Although 12 of 31 variants were not present in Genome Aggregation Database (gnomAD) (11), the minor allele frequency (MAF) for the others ranged from $0.0008 \%$ to $0.16 \%$ (Supplemental Table 2). With 2 exceptions, the identified variants were not homozygous in 123,136 exomes and 15,496 genomes from the gnomAD (accessed October 2019; ref. 11), suggesting that homozygosity for these variants is not tolerated. The 2 exceptions are p.E6K and p.E290Q, which were found in compound heterozygosity with p.R712W in family $\mathrm{C}$ and p.G237C in family $\mathrm{M}$, respectively. The gnomAD contains 42 predicted loss-of-function PLD1 variants (expected number, 62); none of them occurs in the homozygous state, suggesting that $P L D 1$ is intolerant to homozygous loss-of-function variants.

The p.I668F missense variant in PLD1 is a founder variant among Ashkenazi Jews. Given that we found the same homozygous PLD1 variant, p.I668F, in 3 families, we hypothesized that this was a founder variant. Intriguingly, all 3 families with the homozygous PLD1 p.I668F congenital heart defect were of Ashkenazi Jewish ancestry, suggesting a novel founder variant. Ashkenazi Jewish descent was self-reported for family $G$ and determined through principal component analysis (PCA) for families $\mathrm{H}$ and I, which were recruited through the PCGC and found to cluster with an Ashkenazi Jewish reference population (ref. 12 and Supplemental Figure 2). As expected for a founder variant, all homozygous patients shared p.I668F on the background of a common haplotype spanning approximately $881 \mathrm{~kb}$ (chr3:171087469-171969077, hg19). Using the Gamma method (13), we estimated the age of the p.I668F founder variant at 46 generations (95\% CI, 15-154 generations). Assuming a 20-year generational span, a common ancestor with this haplotype would have lived approximately 920 years ago (CI: 300-3080 years). Interestingly, p.I668F has a MAF of approximately $2 \%$ among the Ashkenazi Jewish subpopulation in gnomAD and is rare among the other populations $(0.004 \%-$ $0.14 \%$; ref. 11).

Missense variants in PLD1 lead to reduced enzymatic activity. Placement of the 21 missense variants described above onto the PLD1 structure revealed that they largely clustered in the highly conserved HxKxxxxD (HKD) domains and the C-terminal region (Figure 1B) that are critical for enzymatic activity (14). In contrast, 18 presumed nondeleterious PLD1 missense variants found homozygously in the general population (according to gnomAD, ref. 11; MAF range: $0.001 \%-18 \%$ ) were distributed along the entire length of the protein, including in the N-terminus, PX and $\mathrm{PH}$ domains, and loop regions that are dispensable for catalytic activity (14). Of note, while 5 of 18 variants that were found in the homozygous state in gnomAD resided in the loop region of PLD1, none of the patients carried a variant in the PLD1 loop region (Figure $1 \mathrm{~B}, P=0.017$ ).

To test their effect on PLD1 enzymatic activity, we engineered the disease-associated missense variants into an HA-tagged WT PLD1 expression vector (15) and transfected them into HEK293 cells. Western blot analysis showed levels of expression of the mutant proteins that were comparable to the levels seen for WT PLD1 and PLD1-p.K898R, a previously identified catalytically inactive variant (Supplemental Figure 3 and ref. 16). Enzymatic activity was assessed using an in vitro cellular transphosphatidylation assay in the presence of PMA, which activates PKC, a potent stimulator of PLD1 activity $(15,17)$. PLD1-p.K898R (16) was used as a baseline control (Supplemental Figure 4). We observed a strong to dramatic reduction in PLD1 enzymatic activity for 17 of the 19 variants tested, leading to the prediction that patients carrying biallelic $P L D 1$ variants will generally have less than $25 \%$ residual PLD1 activity in comparison with individuals with WT PLD1 alleles (Figure 2A). Of note, the 2 variants that occurred in the homozygous state in individuals from gnomAD (i.e., p.E6K and p.E290Q) and compound heterozygously in the patients did not affect PLD1 enzymatic activity as measured in this assay.

Since an intact C-terminus is required for enzymatic activity (5, 14), we expected that all of the variants resulting in premature termination of PLD1 protein, such as p.R731Ter/p.R848Ter in the patient with isolated cardiomyopathy from family $\mathrm{T}$, would fully eliminate PLD1 activity. Of note, the other patient who presented with isolated cardiomyopathy (family $\mathrm{U}$ ) was compound heterozygous for 2 missense variants (p.V468D/p.R675Q) that resulted in drastically reduced catalytic activity ( $2.7 \%$ compared with WT). Thus, residual PLD1 enzymatic activity of variants from patients presenting with isolated cardiomyopathy was similar to that of patients who presented with structural cardiac malformations.

PLD1 disease-associated variants localize to sites important for catalytic activity. Some of us recently reported the crystal structure for a catalytically functional PLD1 protein (representing $\sim 60 \%$ of the protein, ref. 5 ) that encompasses most of the diseaseassociated variants reported in this study. Figure $2 \mathrm{~B}$ shows the placement of 18 of the 21 disease-associated missense variants 


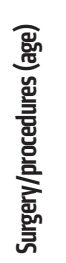

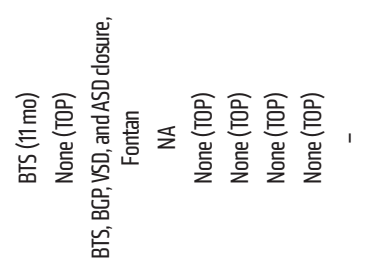

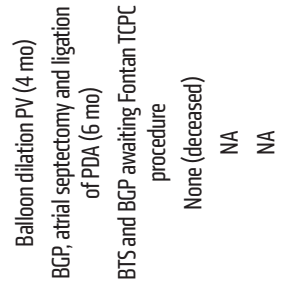

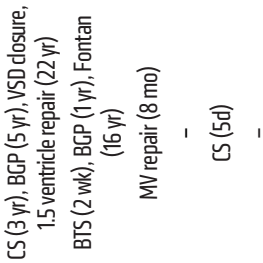

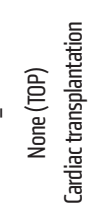

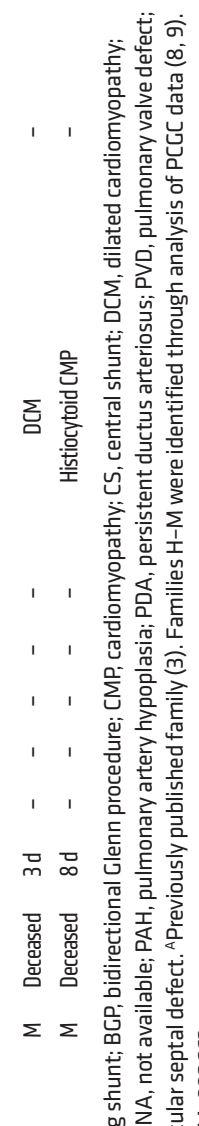

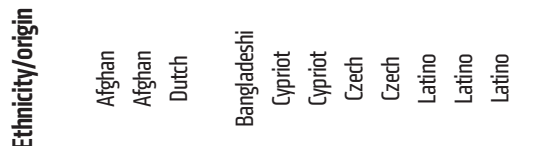

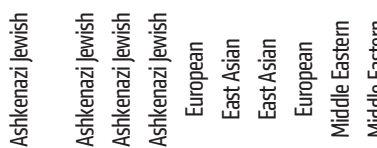

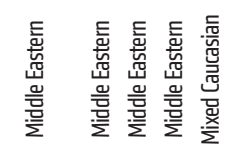

美善晨

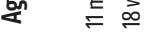

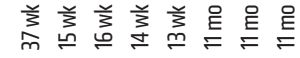

商

部

房产品总

焉

造

变高

吾

จำ

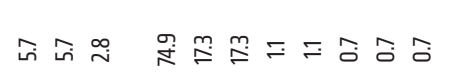

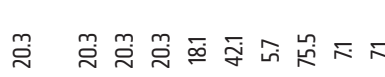

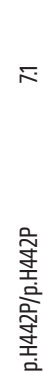

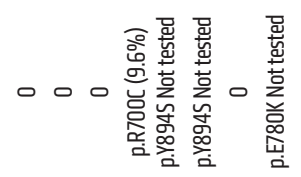

㔛

㔛 亭 $\frac{\pi}{2} \sum_{2}$ (

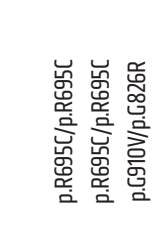

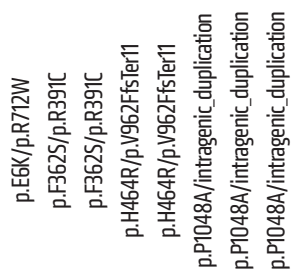

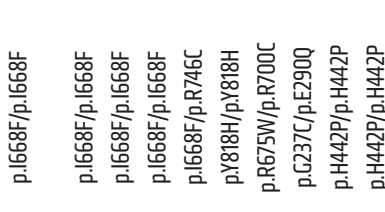

育芒喆

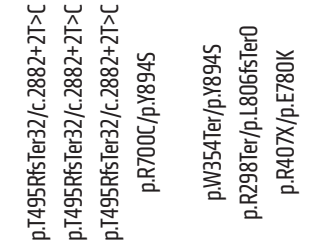

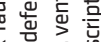

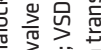

政

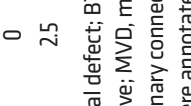

过

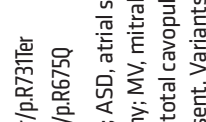

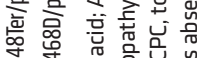

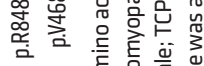

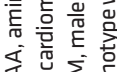

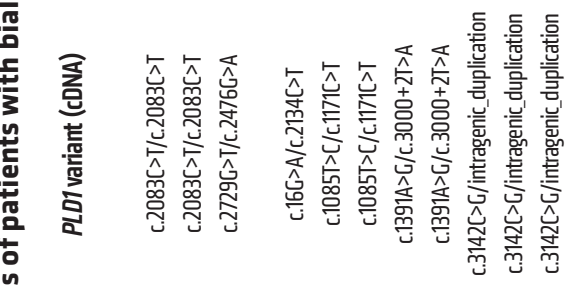
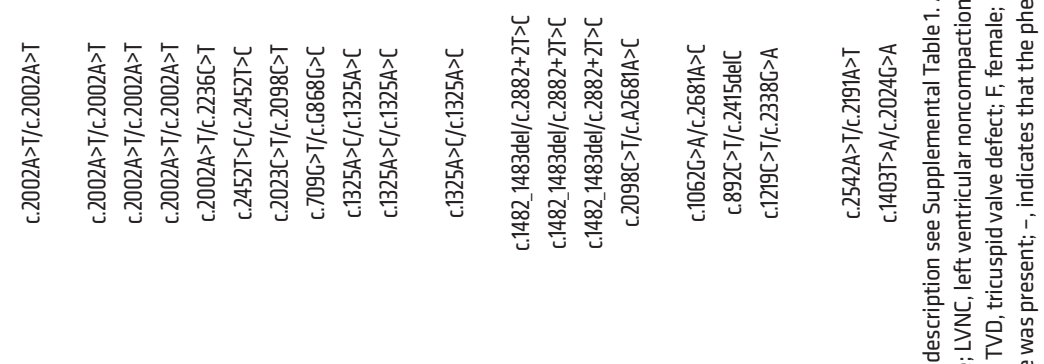

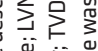

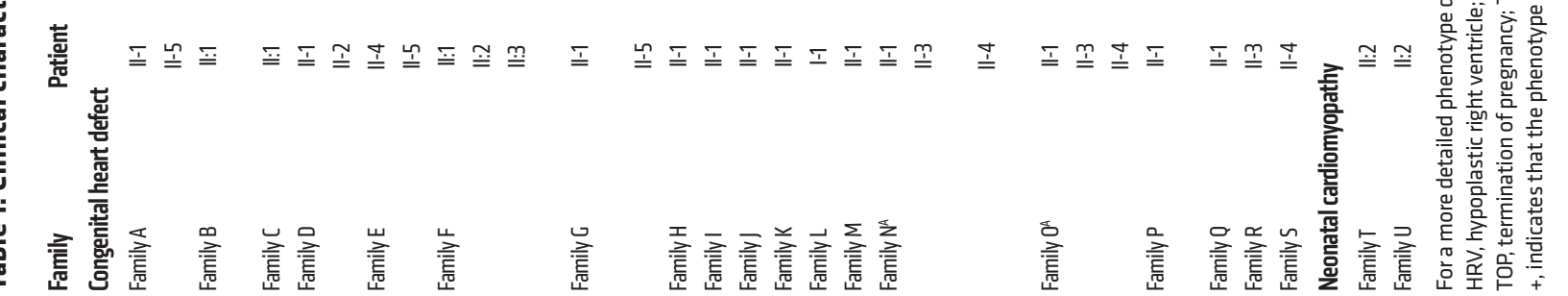


reported here on the 3D structure (see also Supplemental Videos 7-24). Placement of the remaining 3 variants was not assessed, as these were located in the $\mathrm{N}$-terminal (p.E6K) and $\mathrm{PH}$ domain regions (p.E237C and p.E290Q) of the PLD1 protein, which were not included in the crystal structure (5). Strikingly, the majority of the missense variants clustered near sites critical for catalytic activity, which is consistent with the observed reduction of PLD1 enzymatic activity for these variants.

We categorized these variants into 3 groups according to their predicted disruptive effects (Figure $2 \mathrm{~B}$ ). The first class of variants affect the active site of PLD1. These variants either alter a residue directly involved in catalysis (p.H464) or are located within the active site (p.G826, p.Y894, and p.G910), thus affecting catalysis. The second class of variants stabilize elements that form the binding site for the membrane lipid phosphatidylinositol-4,5bisphosphate $\left[\mathrm{PI}(4,5) \mathrm{P}_{2}\right]$, which is a required cofactor for PLD1 activity $(5,15)$. These residues include p.R695, which was mutated in the index family (family A) of our study, as well as p.H442, p.R712, and p.R746. Mutation of these residues are predicted to alter the $\mathrm{PI}(4,5) \mathrm{P}_{2}$ binding site and decrease PLD1 activity. The majority and remaining ones fall into a third class of variants that are distributed throughout the catalytic domain. The mutated residues are buried within the correctly folded structure and are involved in interactions with neighboring residues. These variants would destabilize local interaction networks presumably necessary for proper folding or create new unfavorable interactions. A notable example is the p.I668F founder variant in Ashkenazi Jews. For this variant, substitution with the larger phenylalanine side chain is predicted to create steric clashes with nearby residues that form part of the substrate-binding pocket (Supplemental Video 12). These clashes would alter the shape of the pocket, thus diminishing PLD1 activity.

Only 3 of the variants that we identified in the patients were found outside the catalytic domain. These were located at either the extreme $\mathrm{N}$-terminus (p.E6K) or within the PH domain (p.G237C and p.E290Q), which is required for proper localization of PLD1 to the plasma membrane. As discussed above, both p.E6K (family C) and p.E290Q (family M) seem to be tolerated in the homozygous state and were associated with disease only in combination with p.R712W or p.G237C, respectively. Furthermore, these 2 variants retained full PMA-stimulated enzymatic activity (Figure $2 \mathrm{~A}$ ). In contrast, a $50 \%$ reduction of activity was observed with p.G237C. We predict that this variant may affect membrane binding, as this is the main function of the $\mathrm{PH}$ domain (18), and p.G237 is near 2 residues, C240 and C241, which are posttranslationally modified by palmitoylation to anchor PLD1 to membranes and are functionally required for PLD1's role in regulating exocytosis (18-20).

We also assessed the location of the 18 variants found homozygously in the general population of individuals in gnomAD (11) and found that only 7 of these variants localized to the catalytic domain (Figure 1B and Supplemental Table 3). In contrast to the disease-associated missense variants above that are embedded at sites critical for catalysis, these residues all lie on the periphery of the protein, with their side chains facing outward and having few, if any, interactions with other residues (Supplemental Figure 5). This underscores the importance of evaluating amino acid substitutions in the setting of the 3D structure. In addition, the disease-associated missense variants were more often predicted to be deleterious and affected residues that were more conserved across species (Supplemental Figure 6). Taken together, the disease-associated variants reported here primarily located to sites critical for catalysis and correspondingly exhibited major loss of enzymatic activity.

PLD1 is required for endocardial cell endothelial-mesenchymal transition in atrioventricular cushion explants. Structural atrioventricular (AV) valve defects are the cardinal malformation in patients with PLD1 loss of function. In the embryonic heart, a pivotal early step in valvulogenesis occurs when a subpopulation of endocardial cells overlaying the provalvular cardiac cushions undergoes endothelial-mesenchymal transition (EndoMT) (6). These mesenchymal cells seed the cushions leading to remodeling of the extracellular matrix and contribute to development of the valves and cardiac septa.

To date, many important EndoMT genes have been identified using an in vitro collagen gel assay system (Supplemental Figure 7), in which chick embryo AV cushions (AVCs) are excised, transfected, and explanted endocardial side down onto a collagen pad (21). The explant myocardium compacts and beats while the endocardial cells spread out and form a surface monolayer. Some of the endocardial cells eventually undergo EndoMT, seeding the collagen gel with mesenchymal cells. The spatial specificity observed in vivo, where the AVC, but not the ventricular endocardium, undergoes EndoMT, is reproduced in this in vitro assay (21). We used this assay to investigate the involvement of PLD1 in EndoMT by incubating chick embryo AVC explants with 1-butanol, which suppresses the generation of phosphatidic acid by PLD1, and 3-butanol, which does not suppress phosphatidic acid formation, as a control compound. We observed normal levels of invasion in 3-butanol-treated explants compared with vehicle-incubated controls (our unpublished observations). However, 1-butanol incubation significantly reduced the number of transformed cells compared with the 3-butanol-treated controls (Figure 3A), suggesting that PLD1 function is involved in EndoMT in vitro.

We then confirmed the role of PLD1 using PLD smallmolecule inhibitors (22). PLD1 inhibition dramatically reduced the number of transformed cells, whereas no reduction was seen using an inhibitor of PLD2, the other classic member of the PLD superfamily (Figure 3, B and C). Similarly, siRNA constructs targeting chick PLD1 significantly decreased the number of transformed cells compared with a scrambled control (Figure 3D). We used an siRNA targeting TGF- $\beta$ R3, which is required for EndoMT in AVCs (23), as a positive control. Finally, PLD1 inhibition did not decrease endocardial cell proliferation or increase cell death (Supplemental Figure 8, C and D, and Supplemental Methods, Note 2). Together, these data indicate that PLD1 is required for EndoMT, a pivotal early step in valvulogenesis, and suggest that abnormal EndoMT may underlie, at least in part, the cardiac structural malformations in patients with loss-of-function biallelic PLD1 variants.

\section{Discussion}

We established an international cohort of 30 patients from 21 unrelated families of different ancestries with autosomal- 
A

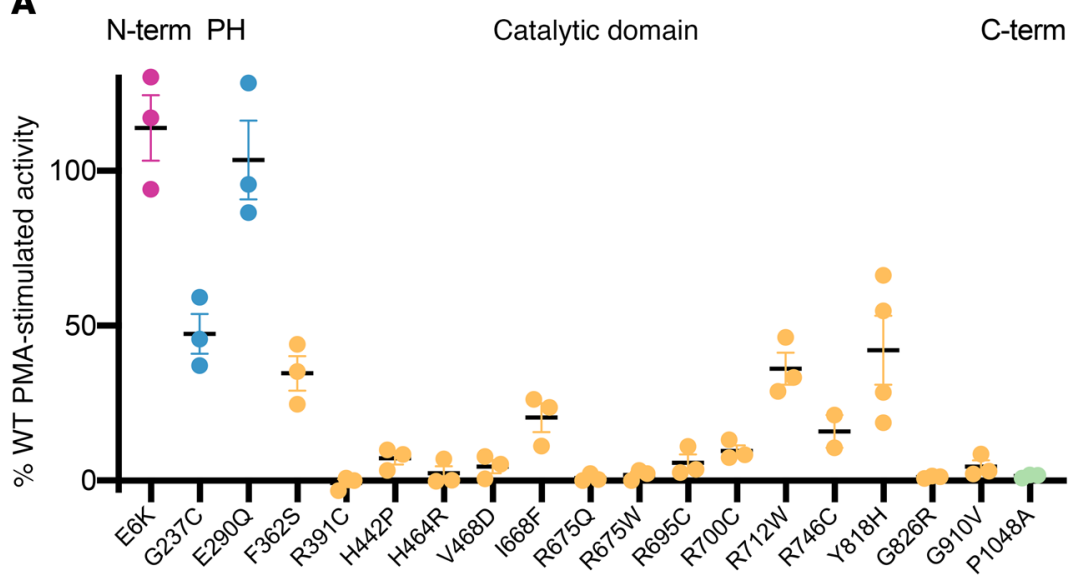

B
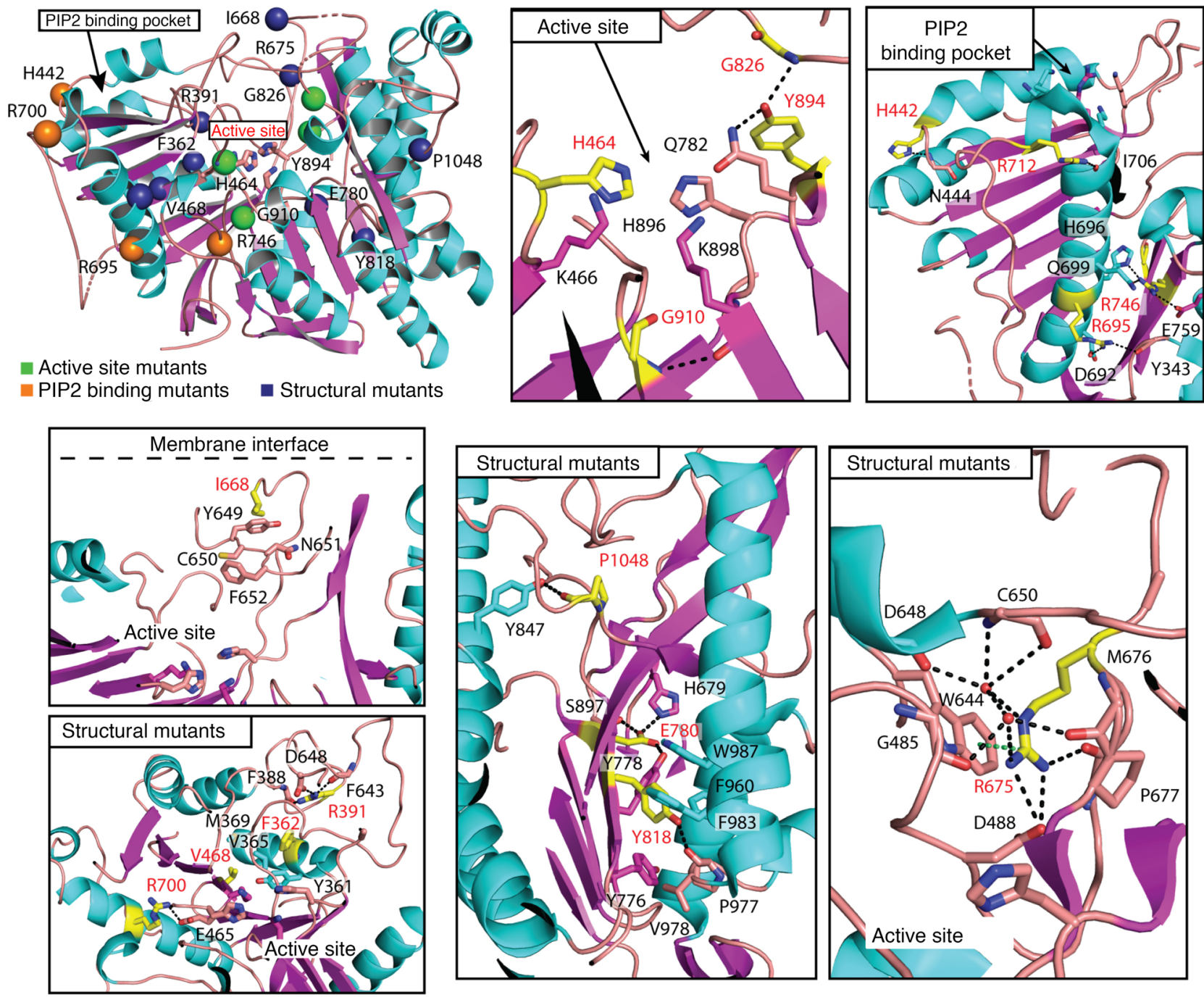

Figure 2. Activity of pathogenic PLD1 variants and their placement on the 3D protein structure. (A) Activity of missense PLD1 variants above the K898R negative control as normalized to the WT PLD1 positive control. Data points represent independent experiments performed in duplicate and averaged. Error bars represent the SEM. Magenta, N-terminus; blue, PH-domain; yellow, catalytic domain; green, C-terminus N-term, N-terminus; C-term, C-terminus. (B) Placement of missense variants on the PLD1 catalytic domain crystal structure. $\alpha$ Helices are shown in cyan, and $\beta$ strands are shown in magenta. Spheres indicate pathogenic missense mutations: green spheres indicate active site variants; orange spheres indicate $\mathrm{PI}(4,5) \mathrm{P}_{2}$-binding $(\mathrm{PIP2}$-binding) mutants; and blue spheres indicate structural mutants. Insets show close-ups of WT residue interactions potentially disrupted by pathogenic variants. Pathogenic residues are represented by yellow sticks; black dashed lines indicate hydrogen bonds; and green dashed lines indicate cation-pi interaction. See Supplemental Videos 7-24 for rotatable presentations and a depiction of the impact of mutations on local structure. 
A

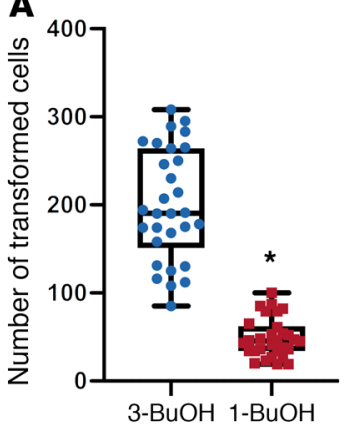

C

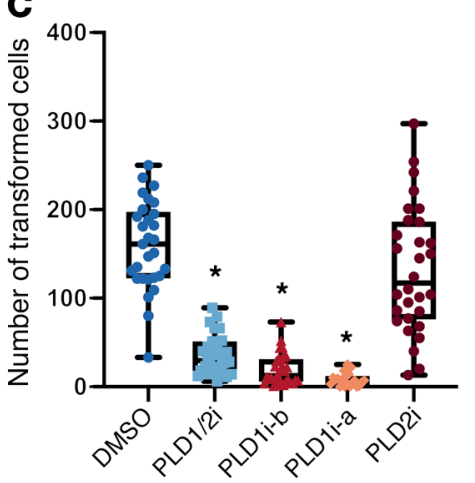

B

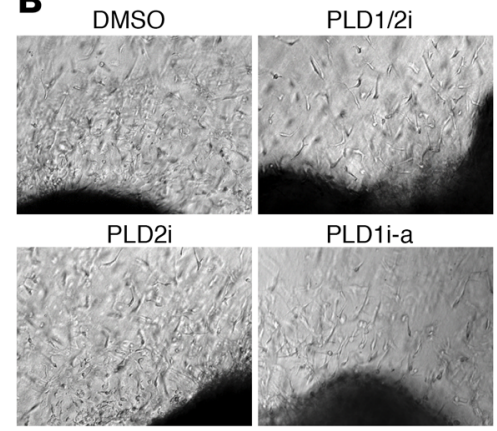

D

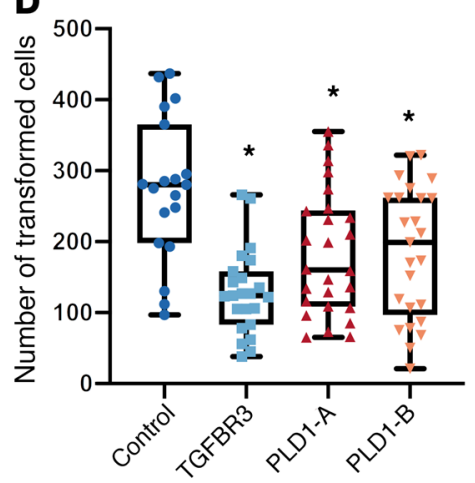

Figure 3. PLD1 is required for EndoMT in AVC explants in vitro. The role of PLD1 in EndoMT was determined using a collagen gel assay by incubating HamburgerHamilton ( $\mathrm{HH}$ ) stage 16 chick AV canal cushion explants on collagen gels for 48 hours with chemical (A), small-molecule inhibitors (B and C) of PLD, or an siRNA (D). The number of cells undergoing EndoMT per explant was counted for each condition over 3 separate experiments. (A) HH16 AVC explants were incubated with either $0.6 \%$ normal butanol (1-BuOH), which inhibits PLD generation of phosphatidic acid, or tertiary butanol (3-BuOH), which does not inhibit phosphatidic acid production. $n=30$ explants for each condition. (B) Representative photomicrographs of AVC explants on collagen gels incubated with $5 \mu \mathrm{M}$ doses of small-molecule inhibitors of PLD1i-a (VU0359595), PLD2i (VU0285655-1), and PLD1/2i (VU0155056, which inhibits both PLD1 and PLD2), or with DMSO. (C) HH16 AVC explants ( $n=30$ for each condition) were incubated with small-molecule PLD inhibitors or DMSO. PLD1/2i (VU0155056) inhibits both PLD1 and PLD2 ( ${ }^{*}=2.9 \times 10^{-17}$ compared with control); PLD1i-B (VU0155069) selectively inhibits PLD1 ( $P=3.9 \times 10^{-20}$ compared with control); PLD2i (VU0285655-1, ${ }^{*} P=0.28$ compared with control); and PLD1i-a (VU0359595, ${ }^{*} P=1.4 \times 10^{-21}$ compared with control). (D) HH16 AVC explants were incubated with siRNAs targeting TGFBR3 $\left({ }^{*} P=2.5 \times 10^{-6}\right.$ compared with control), 2 different regions of $P L D 1-A\left({ }^{*} P=5.7 \times 10^{-3}\right.$ compared with control) and $P L D 1-B\left({ }^{*} P=0.011\right.$ compared with control), or a scrambled siRNA (control). Control explants $(n=19)$ were GC content-matched, randomized siRNA constructs with no homology to any known chick gene. TCFBR3 $(n=23)$ was an siRNA targeting a gene known to be required for EndoMT in vitro. PLD1-A $(n=26)$ and PLD1-B $(n=25)$ were independent constructs targeting different regions of PLD1. All $P$ values were calculated using the Student's $t$ test, and Bonferroni's correction was used to correct for multiple testing. Error bars represent the SEM.

recessive variants in $P L D 1$. This allowed us to expand the phenotypic spectrum of $P L D 1$-associated congenital heart defects and, for the first time to our knowledge, provide evidence that recessive variants in $P L D 1$ can also cause neonatal cardiomyopathy in the absence of congenital heart defects. We demonstrated that the identified missense variants cause a drastic reduction in the catalytic activity of PLD1. By means of pharmacological inhibition of $P L D 1$ and by siRNA knockdown of PLD1 in chick embryo AVC explants, we demonstrated that $P L D 1$ was required for EndoMT, an established pivotal early step in valvulogenesis.

Patients with autosomal-recessive variants in PLD1 predominantly presented with nonsyndromic congenital cardiac valve malformations, primarily affecting the right side of the heart. Specifically, most patients were diagnosed with tricuspid and/or pulmonary valve abnormalities in combination with a hypoplastic right ventricle and hypoplastic pulmonary arteries. While this is in line with the previous report on 2 families with individuals who had PLD1-related autosomal-recessive congenital heart disease (3), in this study, we also identified 5 patients with autosomal-recessive variants in PLD1 who were diagnosed with Ebstein's anomaly. Of note, thus far, the genetic underpinnings of Ebstein's anomaly have remained obscure, with only variants in 2 genes, MYH7 (24) and NKX2-5 (25), being associated with this defect through a candidate-based approach. We further expanded the phenotypic spectrum of PLD1-related disease, as we also identified 2 patients with PLD1 biallelic variants who were diagnosed with severe neonatal cardiomyopathy in the absence of congenital heart defects. Similar to the congenital heart disease cases, both patients with cardiomyopathy carried biallelic variants in PLD1 that are expected to result in severe loss of enzymatic activity. The mechanism underlying this pleotropic effect of PLD1 loss of function, resulting in either valvular heart defects or isolated cardiomyopathy, is currently unknown. It may comprise compensatory mechanisms entailing, among others, modulatory genetics factors, conditions in utero, and stochastic factors. The identification of bialellic PLD1 variants in additional patients with cardiomyopathy in the future will provide further support of causality for this specific phenotype and may enable insights into the underlying mechanism.

Our study robustly implicates recessive genetic variation in PLD1 as a cause of severe cardiac disease. It is expected that consideration of $P L D 1$ as a disease gene will enable reproductive counseling and preimplantation genetic screening in affected families. Although not all parents or siblings carrying a PLD1 variant in the heterozygous state underwent echocardiography, none of them was reported to be clinically affected, suggesting that heterozygous carriers are not at risk for severe congenital heart disease or cardiomyopathy. Notably, in 3 families of Ashkenazi Jewish ancestry, congenital heart defects were caused by homozygosity for the same PLD1 variant, namely p.I668F, which has a MAF of approximately $2 \%$ in this ethnic group. By means of haplotype analysis, we demonstrated that in these 3 families, this variant was inherited from a common ancestor, establishing it as a founder variant in this ethnic group. As this variant is estimated to occur homozygously in up to approximately 1 of 2500 births among Ashkenazi Jews, this finding has clinical implications for assessing the risk of congenital heart defects among individuals of this ancestry (12). As a comparison, a recessive founder variant in GDF1 (MAF of $0.9 \%$ in the Ashkenazi Jewish subpopulation in gnomAD) was recently found to account for approximately $5 \%$ of severe congenital heart defects in Ashkenazi Jews (9). Carriership testing 
for these variants could potentially be used in population-based preconception and prenatal carrier screening to assess the risk of congenital heart defects among descendants of Ashkenazi Jews.

We showed that PLD1 missense variants localized primarily to sites important for catalytic site integrity on the 3D crystal structure of PLD1. Concordantly, we demonstrated that 17 of 19 missense variants led to a drastic reduction in the catalytic activity of PLD1 in an enzymatic assay. Two missense variants, p.E6K (in family C) and p.E290Q (in family M), found in the compound heterozygous state with other variants, showed full PMA-stimulated enzymatic activity. The exact role of these 2 variants in determining the congenital heart defect phenotype in combination with the mutant allele in trans is unclear. Both variants were found once in the homozygous state in approximately 140,000 individuals included in gnomAD (11), which could suggest that these variants do not contribute to the disease or that they are associated with a less severe loss-of-function defect. It should be noted, however, that our enzymatic assay relied on PMA-induced PKC activation of PLD1, and one could hypothesize that these variants might impact stimulation through a different activator (e.g., PLD1 is also stimulated by the Arf and Rho small GTPases at distinct sites on the PLD1 protein, ref. 16) or could alter the subcellular localization or interaction of PLD1 with a downstream effector. Furthermore, we cannot exclude the possibility that the 2 individuals homozygous for these variants in gnomAD were clinically affected, as phenotypic information was not available.

The observation that 10 of 31 variants identified are expected to result in protein truncation suggests that a loss-of-function mechanism underlies the observed cardiac defects. This is further supported by the observations that PLD1 enzymatic activity is drastically reduced by the missense variants and that either pharmacological inhibition or siRNA knockdown of PLD1 in AVC explants inhibited EndoMT. Mechanisms whereby loss of PLD1 activity leads to right-sided AV valve defects could entail established crosstalk between PLD1 signaling and TGF- $\beta$ signaling (26). In the developing heart, TGF- $\beta$ signaling is crucial for the EndoMT that generates the mesenchyme of the cardiac cushions essential for valve formation $(27,28)$. In the adult heart, loss of PLD1 can lead to a reduction in TGF- $\beta$ signaling activity in the context of myocardial infarction (MI) (29), suggesting that PLD1 acts upstream of TGF- $\beta$ signaling. The hearts subjected to myocardial infarction had decreased NF- $\mathrm{BB}$ signaling, which is known to be induced by TGF- $\beta$ (30), and is required for EndoMT in vitro (31). Intriguingly, NF- $\mathrm{KB}$ signaling driven by PLD activity can form an autoregulatory loop that promotes PLD1 expression (32). Downstream mechanisms may also involve sonic hedgehog (Shh) expression, which can be stimulated by TGF- $\beta$ signaling $(33,34)$. Shh maintains secondary heart field cell proliferation, a requirement for normal arterial pole formation (35). Inhibition of Shh signaling at this developmental stage leads to pulmonary atresia. Thus, a reduction in TGF- $\beta$ and SSh signaling resulting from loss-of-function PLD1 variants might underlie the arterial pole defects seen in affected patients.

In the endocardial cushions, EndoMT provides the mesenchymal cells and remodeled matrix essential for the formation of both left and right heart structures, with the inflow cushions contributing to the mitral and tricuspid valves and the outflow cushions contributing to the pulmonary and aortic valves. This "sidedness" offers unique environments with respect to cell populations, extracellular matrix, and hemodynamics, where the mesenchymal cells are instructed in the formation of left- and right-specific structures. These differing contexts may underlie the susceptibility of the right side of the heart to loss of PLD1 activity. In this scenario, cells contributing to the right side of the heart may be less able in this environment to compensate for the disruption of EndoMT in the common cushions. Although no overt structural cardiac defects were noted, mice lacking PLD1 (36) displayed impaired function of the pulmonary and tricuspid valves on echocardiography (3). The mechanism behind the apparent discrepancy in phenotypic severity in humans versus mice is unknown. A potential explanation might involve a compensatory mechanism through upregulation of genes that are able to offset the diminished or loss of function of the mutated PLD1 $(37,38)$.

In conclusion, we have expanded the phenotypic spectrum of PLD1-associated congenital heart defects and provide evidence that recessive variants in PLD1 can also cause neonatal cardiomyopathy in the absence of congenital heart defects. We show that p.I668F is a recessive founder variant among the Ashkenazi Jews. We demonstrated that missense variants in $P L D 1$ predominantly resulted in a strong enzymatic loss of function and that PLD1 is implicated in EndoMT, an established pivotal early step in valvulogenesis, providing a possible mechanism whereby loss of PLD1 function may have led to the cardiac defects observed in the patients studied here. Together, these data provide an increased understanding of disease mechanisms and phenotypic expression associated with genetic defects in PLD1.

\section{Methods}

Case recruitment and DNA sequencing. The index patient in family A was enrolled at the Amsterdam UMC in Amsterdam, the Netherlands. Family B was identified among 75 unrelated patients with severe right-sided valvular congenital heart disease (i.e., tricuspid atresia or stenosis, Ebstein's anomaly, or pulmonary atresia) from the National Registry and DNA bank of congenital heart defects (CONCOR) (7) in the Netherlands. We subsequently used GeneMatcher (4) and an analysis of 2643 congenital heart disease trios (i.e., the affected patient and both parents) from the PCGC who underwent WES $(8,9)$ to identify other patients with biallelic variants in PLD1. Details on case recruitment and next-generation sequencing methods for each family can be found in the Supplemental Methods. Genetic variants were submitted to the NCBI's ClinVar database (www.ncbi.nlm.nih.gov/clinvar/), and the following accession number was obtained: SUB9058450.

cDNA analysis of the c.3000+2T>A PLD1 splicing variant and the proband carrying p.G826R/p.G910V. Total RNA was isolated from PBMCs using TRIzol solution (Thermo Fisher Scientific). RNA concentrations were determined spectrophotometrically at A260 nm by NanoDrop (NanoDrop Technologies), and RNA quality was verified using an Agilent 2100 Bioanalyzer - RNA Lab-On-a-Chip (Agilent Technologies). Aliquots of isolated RNA were stored at $-80^{\circ} \mathrm{C}$ until analysis. First-strand cDNA synthesis was carried out using an oligodT primer and SuperScript III Reverse Transcriptase (Thermo Fisher Scientific). PLD1 cDNA was PCR amplified from the synthesized first-strand cDNA using oligonucleotide primers (Generi Biotech) designed to span and amplify the variant-bearing PLD1 
sequence. The resultant PCR products were analyzed using agarose gel electrophoresis. Individual DNA fragments were extracted from gel slices using the PureLink Quick Gel Extraction Kit (Thermo Fisher Scientific) and sequenced by direct Sanger sequencing as described above. The p.G826R/p.G910V cDNA was subcloned into pcDNA3.1 (Invitrogen, Thermo Fisher Scientific) and Sanger sequenced to determine whether the variants were on the same strand.

Plasmid constructs, cell line, and transfections. We purchased a human PLD1 cDNA clone (BC068976) set in a pGEM-T plasmid (Sino Biological). The clone was fully sequence verified and contained no nucleotide changes. Variants were introduced using site-directed mutagenesis (Q5, New England BioLabs), and the mutant constructs were fully verified by sequencing. Next, the WT and mutant cDNA clones were subcloned into a pcDNA3.1(+) vector for expression and sequence verified. The COS7 cells were cultured according to standard methods. Transfections were performed using polyethylenimine (PEI) in a plasmid to a PEI ratio of 1:4.

In vitro cellular PLD activity. Enzymatic activity was measured on the basis of the ability of PLD1 to catalyze a transphosphatidylation reaction using 1-butanol to generate phosphatidylbutanol (39). Human embryonic kidney 293T (HEK293T) cells were incubated overnight in a 6-well plate and transfected in full media the next day with PLD1 expression plasmids using Fugene (Promega). After overnight transfection for 12-16 hours, the cells were labeled with [ $\left.{ }^{3} \mathrm{H}\right]$-palmitate (ART 129, American Radiolabeled Chemicals) and cultured for 24 hours. The radiolabeled media were then replaced with Opti-MEM and the cells cultured for 1-2 hours prior to the addition of $0.3 \%$ butanol and 100 nM PMA. After 30 minutes, cold methanol was added to stop the reaction, and the lipids were extracted and dried using a speed vacuum and then resuspended in chloroform/methanol (19:1) containing $50 \mu \mathrm{g}$ nonradiolabeled phosphatidylbutanol (Avanti Polar Lipids) to enable location of the labeled enzymatic product on TLC plates (LK5DF, Whatman, MilliporeSigma). The plates were then developed as previously described (39), and the lipids were visualized using iodine flakes (MilliporeSigma) and scraped into $5 \mathrm{~mL}$ scintillation fluid for quantification. In these studies, we used PLD1-K898R as a baseline control. K898 is located in the second of 2 highly conserved HKD motifs (Figure 1B and ref. 15) that constitute key functional elements of the 2 halves of the assembled core catalytic site $(16,40,41)$. A representative experiment showing the production of phosphatidylbutanol (PtdBut) by the WT allele and the absence of its production by the fully inactive K898R allele is shown in Supplemental Figure 4. A limited amount of PtdBut production was observed in the K898R sample, which derives from the small amounts of endogenous WT PLD1 and PLD2 present in the cell line used for the transfections. The percentage of activity of mutant proteins was determined in comparison with the overexpressed WT PLD1 activity after subtraction from the baseline activity observed with the fully inactive K898R mutant allele.

Western blot analysis. HEK293T cells were transfected with $2.5 \mu \mathrm{g}$ mutant HA-tagged PLD1 or WT PLD1 per well in a 6-well plate using Fugene (Promega). After 24-48 hours, the cells were collected in $60 \mathrm{~L}$ homogenization buffer (1 mM DTT, 1 mM EDTA, and $0.1 \mathrm{mM}$ PMSF in PBS) per well and sonicated using a microtip sonicator at the lowest setting. Lysate $(10 \mu \mathrm{L})$ plus $10 \mu \mathrm{L}$ SDS-PAGE loading buffer containing $8 \mathrm{M}$ urea were combined and loaded onto an acrylamide gel for electrophoresis and transfer onto a nylon membrane. The PLD1 protein generated from the transfected expression vector was detected using a monoclonal anti-HA antibody (3F10, MilliporeSigma), followed by secondary goat anti-rat antisera coupled to an infrared dye (IR800, Thermo Fisher Scientific). Images were recorded and bands quantitated using the Odyssey CLx System (LI-COR).

In vitro collagen gel assay. Collagen gel assays (see ref. 21) with siRNA or small molecule additions were performed as described previously (42). siRNA-knockdown experiments have been described elsewhere (43). Briefly, AVCs were excised from stage 16 chick embryo hearts and collected in M199 medium (Mediatech) at room temperature for each experimental condition. For transfection, $4 \mu \mathrm{L}$ siPORT NeoFX (Ambion) was incubated in a final volume of 100 $\mu \mathrm{L}$ M199 for 10 minutes at room temperature. Next, the appropriate final concentration (for $300 \mu \mathrm{L}$ total volume) of the siRNA was added to a final volume of $100 \mu \mathrm{L}$. These 2 tubes were mixed and incubated for 15 minutes at room temperature to allow complexes to form. The $200 \mu \mathrm{L}$ mixture was then added to the $100 \mu \mathrm{L}$ containing AVCs, and this solution was incubated at $37^{\circ} \mathrm{C}, 5 \% \mathrm{CO}_{2}$ for $45 \mathrm{~min}-$ utes. Explants were then placed endothelial side down on collagen gels $(1.86 \mathrm{mg} / \mathrm{mL})$ and incubated under the same conditions for 48 hours prior to fixation $(0.8 \%$ formaldehyde, $0.05 \%$ glutaraldehyde for 5 minutes at room temperature). Controls for siRNA toxicity were randomized genomic DNA base pair composition-matched (\%GC base pair matched) constructs that did not correspond to any sequence in the chick genome. The following antisense strand siRNAs were used: PLD1-A, antisense strand siRNA, AUGGUGUACACGUUGAGGCtt and PLD1-B, antisense strand siRNA, CUUAGCGUUCACAUACCACtt. The positive control was an siRNA targeting Tgfbr3 (44). For experiments using small-molecule inhibitors, AVCs were harvested and placed endothelial side down on collagen gels in the presence of the indicated molecule concentrations. Explants were incubated for 48 hours prior to fixation as above. After incubation, EndoMT was quantified by counting the number of cells with mesenchymal morphology that invaded the collagen gel.

PCA to define the ancestry of PCGC congenital heart disease cases. PCAs were performed in PLINK 2.0 (45) on the WES PCGC cases, as previously described (46), using data from the 1000 Genomes populations (Phase 3v5) and on the Ashkenazi Jewish individuals from the NCBI's Gene Expression Omnibus (GEO) database (GEO GSE23636; ref. 12) as a reference. To increase the genetic overlap with the WES PCGC cases and the 1000 Genomes populations, we performed genome-wide imputation using Eagle2 phasing, Minimac3, and the Haplotype reference consortium (HRCr1.1) panel implemented on the Michigan Imputation Server for the data set for Ashkenazi Jewish individuals. After imputation, we performed stringent genetic quality control using hard genotype calls after imputation (genotype probability $>0.9$ ) and restricted to variants with an imputation score above 0.8. We excluded variants with a genotype missingness greater than 0.01, a Hardy-Weinberg equilibrium test at a $P$ value of less than 0.05 , a phenotype-biased missing test at a $P$ value of less than 0.05 , and a MAF of less than 0.05. Downstream analyses were conducted in $\mathrm{R}$, version 3.4.3.

Haplotype analysis and variant dating. Haplotypes were generated using WES data. Estimation of the p.I668P variant age was conducted using the Gamma method (13). This method uses the genetic length of ancestral haplotypes shared between individuals carrying the variant and has as a major advantage that it can be reliably applied to small samples with high-density SNP data. 
Statistics. All error bars represent the SEM. Comparisons between 2 groups were performed using the 2-tailed Student $t$ test for normally distributed continuous variables, the Mann-Whitney $U$ test for non-normally distributed continuous variables, and the $\chi^{2}$ or Fisher's exact test, as suitable, for categorical variables. A $P$ value of less than 0.05 was considered significant. For multiple comparisons, Bonferroni's correction for multiple testing was applied. Statistical analyses were performed with GraphPad Prism (version 7.02, GraphPad Software) and $\mathrm{R}$ (version 4.0.0).

Study approval. The study protocol was approved by the local IRBs where the patients were followed, and signed informed consent was obtained from the patients or their parents. The IRBs' institutions and locations are as follows: family A: Amsterdam UMC, Amsterdam, the Netherlands; family B: National Registry and DNA bank of congenital heart defects, the Netherlands; family C: Bambino Gesù Children's Hospital IRCCS, Rome, Italy; family D: The Cyprus Institute of Neurology and Genetics, Nicosia, Cyprus; family E: Charles University and General University Hospital, Prague, Czech Republic; family F: University of Minnesota, Minneapolis, Minnesota, USA; family G, N, and O: Hebrew University Medical Center, Jerusalem, Israel. family P: UPMC Children's Hospital of Pittsburgh, Pittsburgh, Pennsylvania, USA; family Q: Université Bourgogne-Franche Comté, Dijon, France; family R: University of Groningen, University Medical Center Groningen, Groningen, the Netherlands; family S: D Ann \& Robert H. Lurie Children's Hospital of Chicago, Illinois, USA; family T: Leiden University Medical Center, Leiden, the Netherlands; and family U: Ochsner Clinic Foundation, New Orleans, Louisiana, USA. Families $\mathrm{H}-\mathrm{M}$ were recruited to the Congenital Heart Disease Network Study of the Pediatric Cardiac Genomics Consortium (CHD GENES: ClinicalTrials.gov identifier NCT01196182), and the study protocol was approved by the local IRB.

\section{Author contributions}

CRB, MAF, and NL designed and supervised the study. NL and MAF compiled results, wrote the manuscript, and prepared all the figures. NL, VS, and DZ performed sequencing data analysis. NL, SAC, FT, DSM, ATS, ZP, JYR, SCB, HM, MA, TM, JJ, DMN, AR, AAK, GT, BAL, ADJTH, CF, DO, GTRM, ACD, KB, MM, FCR, $\mathrm{MH}, \mathrm{MCH}, \mathrm{ADJTH}, \mathrm{BM}, \mathrm{SK}, \mathrm{GWES}$, JCH, VT, OE, BW, ASDP, $\mathrm{KHK}$, and SC recruited patients and gathered detailed clinical information for the study. NL and SAB reviewed and summarized patients' characteristics. PDK and RDL performed multiplex PCR sequencing followed by NGS in patient 1 from family A. ACD performed the autopsy of a patient. CMS performed the Western blots and PLD activity and localization assays. NL, AVP, and EML performed haplotype analyses. NL performed PCAs. MH and QG assessed PLD1-knockout hearts. MAF was involved in experimental design and manuscript preparation. MVA and FZB analyzed the PLD1-mutant structural data. DMDL, JYR, and JVB performed and analyzed the explant assays. SK and LP performed RT-PCR analysis of mRNA. LB and AI generated PLD1-mutant plasmids. All the authors read and agreed on the final draft of the manuscript. The order of the co-first authors was assigned on the basis of their relative contributions to the study.

\section{Acknowledgments}

We thank the families for their participation and collaboration. CRB, AVP and NL acknowledge the support from the Dutch Heart Foundation (CVON 2018-30 PREDICT2 and CVON201418 CONCOR-GENES to CRB), the Netherlands Organization for Scientific Research (VICI fellowship, 016.150.610, to CRB), the Children's Heart Foundation (to CRB), and the PROCEED project funded under the ERA PerMed Joint Translational Call Initiative (to $\mathrm{CRB}$ ). NL acknowledges support from the CONCOR-GENES Young Talent Program. This work was supported by NIH grants R35GM128666 (to MVA), T32GM092714 (to FZB), R01GM084251 (to MAF), and U54092551 (to DMD, JYR, and JVB) and National Science Foundation (NSF) grant 1612689 (to CMS). SK, VS, and LP were supported by the Ministry of Health of the Czech Republic (NV19-07-00136) and by institutional programs of the Charles University in Prague (UNCE/MED/007, SVV2016/260148 and PROGRES-Q26/LF1). We thank The National Center for Medical Genomics (LM2018132) for instrumental and technical support with the sequencing analysis (to SK, VS, and LP). The authors thank Daoud Sie (Amsterdam UMC, Amsterdam, the Netherlands) for advice on analyses of PCGC WES data. A subset of the WES data was generated by the PCGC under the auspices of the NHLBI's Bench to Bassinet Program (https://benchtobassinet.com) (dbGaP study accession number phs001194.v2.p2). The PCGC program is funded by the National Heart, Lung, and Blood Institute (NHLBI), NIH, US Department of Health and Human Services, through grants UM1HL128711, UM1HL098162, UM1HL098147, UM1HL098123, UM1HL128761, and U01HL131003. This manuscript was not prepared in collaboration with investigators of the PCGC, has not been reviewed or approved by the PCGC, and does not necessarily reflect the opinions of the PCGC investigators or the NHLBI.

Address correspondence to: Connie R. Bezzina or Najim Lahrouchi, Amsterdam UMC, AMC Heart Center, Department of Clinical and Experimental Cardiology, Meibergdreef 9, 1105 AZ Amsterdam, Netherlands. Phone: 31.20.566.5403; Email: c.r.bezzina@ amsterdamumc.nl (CRB); n.lahrouchi@amsterdamumc.nl (NL). Or to: Michael A Frohman, Department of Pharmacological Sciences, 438 CMM, Stony Brook University, Stony Brook, New York 11794, USA. Phone: 631.632.1476; Email: Michael.Frohman@ stonybrook.edu.
1. Dolk H, et al. Congenital heart defects in Europe: prevalence and perinatal mortality, 2000 to 2005. Circulation. 2011;123(8):841-849.

2. Nelson RK, Frohman MA. Physiological and pathophysiological roles for phospholipase D. J Lipid Res. 2015;56(12):2229-2237.

3. Ta-Shma A, et al. Congenital valvular defects associated with deleterious mutations in the PLD1 gene. JMed Genet. 2017;54(4):278-286.
4. Sobreira N, et al. GeneMatcher: a matching tool for connecting investigators with an interest in the same gene. Hum Mutat. 2015;36(10):928-930.

5. Bowling FZ, et al. Crystal structure of human PLD1 provides insight into activation by $\mathrm{PI}(4,5) \mathrm{P}_{2}$ and RhoA. Nat Chem Biol. 2020;16(4):400-407.

6. Barnett JV, Desgrosellier JS. Early events in valvulogenesis: a signaling perspective. Birth Defects Res Part C Embryo Today Rev.
2003;69(1):58-72.

7. Vander Velde ET, et al. CONCOR, an initiative towards a national registry and DNA-bank of patients with congenital heart disease in the Netherlands: rationale, design, and first results. Eur J Epidemiol. 2005;20(6):549-557.

8. Gelb B, et al. The Congenital Heart Disease Genetic Network Study: rationale, design, and early results. Circ Res. 2013;112(4):698-706. 
9. Jin SC, et al. Contribution of rare inherited and de novo variants in 2,871 congenital heart disease probands. Nat Genet. 2017;49(11):1593-1601.

10. van der Palen RLF, et al. Uhl's anomaly: clinical spectrum and pathophysiology. Int J Cardiol. 2016;209:118-121.

11. Karczewski KJ, et al. The mutational constraint spectrum quantified from variation in 141,456 humans. Nature. 2020;581(7809):434-443.

12. Bray SM, et al. Signatures of founder effects, admixture, and selection in the Ashkenazi Jewish population. Proc Natl Acad Sci U S A. 2010;107(37):16222-16227.

13. Gandolfo LC, et al. Dating rare mutations from small samples with dense marker data. Genetics. 2014;197(4):1315-1327.

14. Sung TC, et al. Structural analysis of human phospholipase D1. J Biol Chem. 1999;274(6):3659-3666.

15. Hammond SM, et al. Human ADP-ribosylation factor-activated phosphatidylcholinespecific phospholipase $\mathrm{D}$ defines a new and highly conserved gene family. J Biol Chem. 1995;270(50):29640-29643.

16. Sung TC. Mutagenesis of phospholipase D defines a superfamily including a trans-Golgi viral protein required for poxvirus pathogenicity. EMBO J. 1997;16(15):4519-4530.

17. Sung TC, et al. Structural analysis of human phospholipase D1. J Biol Chem. 1999;274(6):3659-3666.

18. Du G, et al. Regulation of phospholipase D1 subcellular cycling through coordination of multiple membrane association motifs. JCell Biol. 2003;162(2):305-315.

19. Vitale N, et al. Phospholipase D1: a key factor for the exocytotic machinery in neuroendocrine cells. EMBO J. 2001;20(10):2424-2434.

20. Sugars JM, et al. Fatty acylation of phospholipase D1 on cysteine residues 240 and 241 determines localization on intracellular membranes. J Biol Chem. 1999;274(42):30023-30027.

21. DeLaughter DM, et al. What chick and mouse models have taught us about the role of the endocardium in congenital heart disease. Birth Defects Res A Clin Mol Teratol. 2011;91(6):511-525.
22. Lavieri RR, et al. Design, synthesis, and biological evaluation of halogenated N-(2-(4-oxo-1phenyl-1,3,8-triazaspiro[4.5]decan-8-yl)ethyl) benzamides: discovery of an isoform-selective small molecule phospholipase D2 inhibitor. J Med Chem. 2010;53(18):6706-6719.

23. Bassing $\mathrm{CH}$, et al. A transforming growth factor beta type I receptor that signals to activate gene expression. Science. 1994;263(5143):87-89.

24. Postma AV, et al. Mutations in the sarcomere gene MYH7 in Ebstein anomaly. Circ Cardio vasc Genet. 2011;4(1):43-50.

25. Benson DW, et al. Mutations in the cardiac transcription factor NKX2.5 affect diverse cardiac developmental pathways. JClin Invest. 1999;104(11):1567-1573.

26. Piazza GA, et al. Lysophosphatidic acid induction of transforming growth factors $\alpha$ and $\beta$ : modulation of proliferation and differentiation in cultured human keratinocytes and mouse skin. Exp Cell Res. 1995;216(1):51-64.

27. Xu J, et al. TGF- $\beta$-induced epithelial to mesenchymal transition. Cell Res. 2009;19(2):156-172.

28. Gise A von, Pu WT. Endocardial and epicardial epithelial to mesenchymal transitions in heart development and disease. Circ Res. 2012;110(12):1628.

29. Schönberger T, et al. Pivotal role of phospholipase D1 in tumor necrosis factor- $\alpha$-mediated inflammation and scar formation after myocardial ischemia and reperfusion in mice. Am J Pathol. 2014;184(9):2450-2464.

30. Sakurai H, et al. Functional interactions of transforming growth factor $\beta$-activated kinase 1 with $\mathrm{I} \kappa \mathrm{B}$ kinases to stimulate NF- $\kappa \mathrm{B}$ activation. J Biol Chem. 1999;274(15):10641-10648.

31. DeLaughter DM, et al. Spatial transcriptional profile of the chick and mouse endocardial cushions identify novel regulators of endocardial EMT in vitro. J Mol Cell Cardiol. 2013;59:196-204.

32. Kang DW, et al. Autoregulation of phospholipase $\mathrm{D}$ activity is coupled to selective induction of phospholipase D1 expression to promote invasion of breast cancer cells. Int J Cancer. 2011;128(4):805-816.

33. Javelaud $D$, et al. TGF- $\beta /$ SMAD/GLI2 signaling axis in cancer progression and metastasis. Cancer Res. 2011;71(17):5606-5610.

34. Dennler $\mathrm{S}$, et al. Induction of sonic hedgehog mediators by transforming growth factor- $\beta$ : Smad3-dependent activation of Gli2 and Gli1 expression in vitro and in vivo. Cancer Res. 2007;67(14):6981-6986.

35. Dyer LA, Kirby ML. Sonic hedgehog maintains proliferation in secondary heart field progenitors and is required for normal arterial pole formation. Dev Biol. 2009;330(2):305-317.

36. Elvers $\mathrm{M}$, et al. Impaired $\alpha(\mathrm{IIb}) \beta(3)$ integrin activation and shear-dependent thrombus formation in mice lacking phospholipase D1. Sci Signal. 2010;3(103):ra1.

37. El-Brolosy MA, et al. Genetic compensation triggered by mutant mRNA degradation. Nature. 2019;568(7751):193-197.

38. Ma Z, et al. PTC-bearing mRNA elicits a genetic compensation response via Upf3a and COMPASS components. Nature. 2019;568(7751):259-263.

39. Morris AJ, et al. Measurement of phospholipase D activity. Anal Biochem. 1997;252(1):1-9.

40. Leiros I, et al. The first crystal structure of a phospholipase D. Structure. 2000;8(6):655-667.

41. Dixon JE, Stuckey JA. Crystal structure of a phospholipase D family member. Nat Struct Biol. 1999;6(3):278-284.

42. Townsend TA, et al. Endocardial cell epithelialmesenchymal transformation requires Type III TGF $\beta$ receptor interaction with GIPC. Cell Signal. 2012;24(1):247-256.

43. Townsend TA, et al. Transforming growth factor-beta-stimulated endocardial cell transformation is dependent on Par6c regulation of RhoA. J Biol Chem. 2008;283(20):13834-13841.

44. Townsend TA, et al. BMP-2 and TGF $\beta 2$ shared pathways regulate endocardial cell transformation. Cells Tissues Organs. 2011;194(1):1-12.

45. Chang CC, et al. Second-generation PLINK: rising to the challenge of larger and richer datasets. Gigascience. 2015;4(1):7.

46. Belkadi A, et al. Whole-exome sequencing to analyze population structure, parental inbreeding, and familial linkage. Proc Natl Acad Sci U S A. 2016;113(24):6713-6718. 